\title{
The Top 100 Most Frequently Cited Publications Concerning Anti-PD-I/PD-LI Therapy for Lung Cancer: A Bibliometric Analysis
}

This article was published in the following Dove Press journal: Cancer Management and Research

Yang $\mathrm{Li}^{\prime}$

Moyang $\mathrm{Lv}^{2}$

Jingting $\mathrm{Liu}^{3}$

Jun $\mathrm{Ma}^{4}$

Man Liang ${ }^{5, *}$

$\mathrm{Na}$ Zheng ${ }^{6, *}$

'Department of Thoracic Surgery, The First Hospital of jilin University, Changchun, People's Republic of China: ${ }^{2}$ Grade 2018 Clinical Medicine, Army Medical University, Chongqing, People's Republic of China; ${ }^{3}$ Grade 2017 Nursing, Shenzhen University Health Science Center, Shenzhen, People's Republic of China; ${ }^{4}$ Department of Radiology, Affiliated Hospital of Beihua University, Jilin, People's Republic of China; ${ }^{5}$ Department of Forensic Science, Tongji Medical College, Huazhong University of Science \& Technology, Wuhan, People's Republic of China; ${ }^{6}$ Department of Pathology, Shenzhen University Health Science Center, Shenzhen, People's Republic of China

*These authors contributed equally to this work
Background: Globally lung cancer is one of the most common cancers, and is responsible for almost $20 \%$ of all cancer care costs. As a potential treatment for lung cancer, anti-PD-1/ PD-L1 therapy has become a novel scientific hotspot in recent decades. The present study aims at exploring the status and trends of the top frequently cited publications about the antiPD-1/PD-L1 therapy for lung cancer via bibliometric analysis.

Methods: The publications concerning anti-PD-1/PD-L1 therapy for lung cancer were searched on the core collection database of Web of Science, setting the time period for retrieval from 1950 to 2019 . The top 100 most frequently cited publications were retrieved, and the bibliometric data were mainly accessed through an open online analysis platform and VOSviewer software.

Results: The cited frequencies about the top 100 cited publications ranged from 218 to 6248. These articles were published in 39 publications, which were mainly ranked in Q1. The top journal in terms of the number of the articles was the New England Journal of Medicine (16 articles). The most frequently nominated author was Brahmer, JR from Sidney Kimmel Comprehensive Cancer Center, while the most contributing institution was Memorial Sloan Kettering Cancer. The United States acted as the pioneer in this new field of research and led plentiful of national and international co-operations. Immunotherapy, nivolumab, cell lung-cancer, safety, and docetaxel appeared more frequently as keywords.

Discussions: To sum up, high quality journals, influential authors and institutions and research with high quality evidence were apt to attract more attention and possess more public credibility. Moreover, the bibliometric analysis is yielding up its advantage of identifying and analyzing the characteristics and changes in the intellectual structures of a special topic.

Keywords: anti-PD-1/PD-L1 therapy, bibliometrics, lung cancer, VOSviewer

\section{Introduction}

Cancer ranks as the second or even the first cause of premature death in most countries, ${ }^{1}$ for instance, lung cancer is persistently the leading cause of cancer death both in males and females. Therefore, as a disease, it contributes substantially to the global burden and health care costs, and is responsible for almost $20 \%$ of all cancer care costs. ${ }^{2}$ Many endeavors focus on the prevention, screening, treatment, and palliation to curb the economic burden related to lung cancer. ${ }^{1,3}$ Fortunately, the improvements in treatment and early detection, as well as declines in smoking, have resulted in a continuous decline in the lung cancer death rate in recent decades. ${ }^{4}$
Correspondence: Man Liang Medical College, Huazhong University of Science \& Technology, Wuhan, People's Republic of China

Email liangman@hust.edu.cn

$\mathrm{Na}$ Zheng

Department of Pathology, Shenzhen University Health Science Center Shenzhen, People's Republic of China Email19629194@qq.com 
Important advancements in the treatment of lung cancer have been achieved over the past two decades, increasing the understanding of the mechanisms of tumor progression, or tumor escape.

Immunotherapies of lung cancer, which are developed mainly based on the mechanisms of tumor escape, manipulate the immune system to reactivate the antitumor immune response and to overcome the pathways leading to escape. Multiple mechanisms have been described to account for the evolution of this escape, the most attractive one is upregulating the expression of immune checkpoint proteins. ${ }^{5}$ Programmed cell death protein 1 (PD-1, or B7-H1) is a costimulation receptor expressed by activated $\mathrm{T}$ cells, and binds to its ligand programmed death ligand 1 (PD-L1) leading to a transient or permanent inhibition of CD8+ T cells cytotoxic properties. PD-L1 protein expressions and tumor mutational burden have emerged as the most well-validated biomarkers in amount of lung cancer patients. Therefore, as a significant part of immune checkpoint inhibitors therapy, anti-PD-1/PD-L1 therapy, can help the immune system fight cancer. Over the past few decades, anti-PD-1/PD-L1 therapy has developed targeting cancer at a striking rate. Continued research into novel first-line and second-line drugs and combination therapies have expanded the clinical benefit to a broader population and improved outcomes in non small cell lung cancer (NSCLC). ${ }^{6}$ Even though, significant overall survival benefits with the addition of PD-L1 antibody to standard chemotherapy have been shown in the treatment of extensive stage small cell lung cancer (SCLC), the overall benefit of immunotherapy in SCLC remains somewhat disappointing in comparison with the results seen in NSCLC. ${ }^{7}$ Currently, there are several PD-1/PD-L1 blocking agents available in clinics, and immunotherapy-based regimen alone or in combination with chemotherapy is now the preferred option, including nivolumab, pembrolizumab, and atezolizumab. ${ }^{8}$ Evidence shows that the use of immunotherapy has led to unprecedented survival benefits especially compared to treatment with chemotherapy alone in selected patients. The breakthroughs in immunotherapy have greatly benefited a subset of lung cancer patients, not only the NSCLC, but also the extensive-stage SCLC, ${ }^{9}$ and the most important is that they are bringing forth a paradigm shift in the drugs approved for cancer treatment ${ }^{10}$ Antibody-drug conjugate therapy, another anti-PD-1/PD-L1 therapy, has been widely concerned. Antitumor activity of lung cancer has been accomplished by conjugating antibodies with different effector molecules that accomplish cell death after antibody binding and internalisation. As an effective antiPD-1/PD-L1 therapy, growing clinical application highlights the importance of the recognition and management of its unique toxicity profile in lung cancer patients. Involving the dermatologic toxicity, gastrointestinal toxicity, endocrine toxicity, pneumonitis, and rheumatologic toxicity, the adverse effects of anti-PD-1/PD-L1 therapy of lung cancer have been recognized recently. ${ }^{5}$

Anti-PD-1/PD-L1 therapy is important in treating lung cancer, but there is no bibliometric analysis on researches in this field. The purpose of our study is to use bibliometric methods to analyze the top 100 most cited articles on anti-PD-1/PD-L1 therapy for lung cancer, hoping to have a better understanding of current status and trend of those researches via analyzing their characteristics, and to summarize the reasons of high citation.

\section{Materials and Methods Data Sources}

Publications concerning anti-PD-1/PD-L1 therapy for lung cancer were retrieved from the Core Collection Database of the Science Citation Index in Web of Science of from 1950 to 2019 (updated to March 3, 2020). The search strategy was that combined the terms related to "lung cancer" and "anti-PD-1/ PD-L1," shown in the Supplementary Table S1. The inclusion criteria were as follows: (a) publications were written in English; (b) the types of publications were original articles and reviews. Retrieved publications were arranged in descending order according to the cited frequencies, and the top 100 most cited publications were finally pooled. Two independent reviewers separately evaluated the retrieved scientific works to identify those that were concerned with antiPD-1/PD-L1 therapy for lung cancer. If there were different opinions, a third reviewer would be consulted, and consensus was finally achieved through discussion.

\section{Data Extraction}

The top 100 most frequently cited articles were retrieved, and the following information was extracted: the authors, the affiliations of the first authors and the corresponding authors, countries, the years of publication, the cited frequencies, article title, article types, journals, key words, and impact factor of the journals (2018 edition of Journal Citation Reports).

\section{Statistical Analysis}

The data were imported in Microsoft Excel 2007 software to descriptive statistical analyse, including those on publication year, authors, the affiliations, countries, journals, cited frequencies, etc. Apart from the Excel software, the 
bibliometric analysis were performed based on the online platform (https://bibliometric.com) and VOSviewer 1.6.14, which were applied to construct the bibliographic coupling network based on key words and co-authorship relations, so as to implement network visualization analysis.

\section{Results}

\section{Characteristics of Included Studies}

A total of 4,976 publications were retrieved from the Core Collection Database of Web of Science concerning to antiPD-1/PD-L1 therapy for lung cancer, and the flow diagram for screening was shown in Figure 1. All of the 100 most frequently cited publications were published between 2002 and 2018, from 0 to 27 articles each year, and 86\% were published between 2014 and 2018. Of the publications, 81 were articles and 19 were reviews. These scientific works were published by researchers from 10 countries, and most were from the United States, France, Germany, Spain,

\section{Study flow diagram}

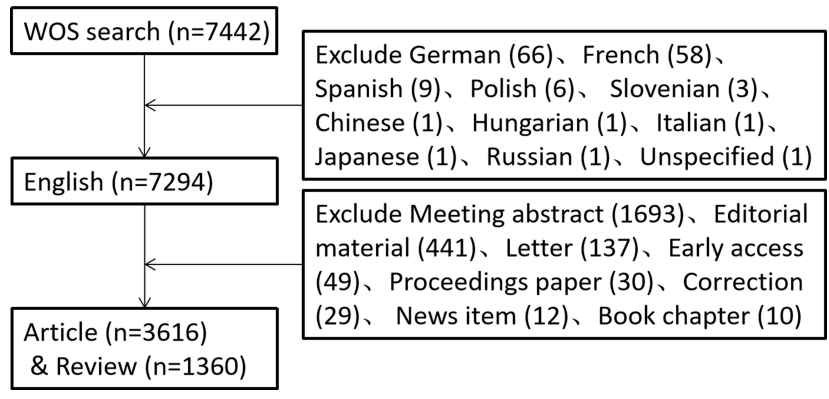

Figure I The study flow diagram for screening. and Italy (Figure 2). They were published in 39 journals, and the impact factors ranged from 2.776 to 70.67 based on the 2018 edition of Journal Citation Reports, and the basic information of the journals is shown in Table 1. The average and median cited frequencies, range of citation number, and interquartile range of the 100 articles were 736, 368.5, 218 to 6248 , and 268.5 , respectively. The information of these scientific works was listed in Supplementary Table S2.

\section{The Top 10 Authors}

Among the 100 publications, the researcher who published most articles was Brahmer JR from Sidney Kimmel Comprehensive Cancer Center, USA, reaching a total of 11 publications, while the second author Pardoll DM came from the same institution (Table 2). Among the top 10 authors, all of them were from United States. Moreover, only three authors, Hellmann MD, Wolchok JD, and Rizvi NA, contributed to the research "Mutational landscape determines sensitivity to PD-1 blockade in non-small cell lung cancer," 11 while another seven authors all contributed to the most frequently cited research "Safety, activity, and immune correlates of Anti-PD-a antibody in cancer." ${ }^{\prime 2}$ All the authors had the H-index of at least 45, which meant that at most 45 of all one' papers were cited at least 45 times. It is a certain that an higher h-index means the greater effect of publications.

\section{The Top 10 Institutions}

Memorial Sloan Kettering Cancer Center (Mem Sloan Kettering Canc Ctr) contributed to $45 \%$ of the top 100 cited articles, followed by The University of Texas MD Anderson Cancer Center (Univ Texas MD Anderson Can Ctr, 29\%), and Johns Hopkins University (Johns

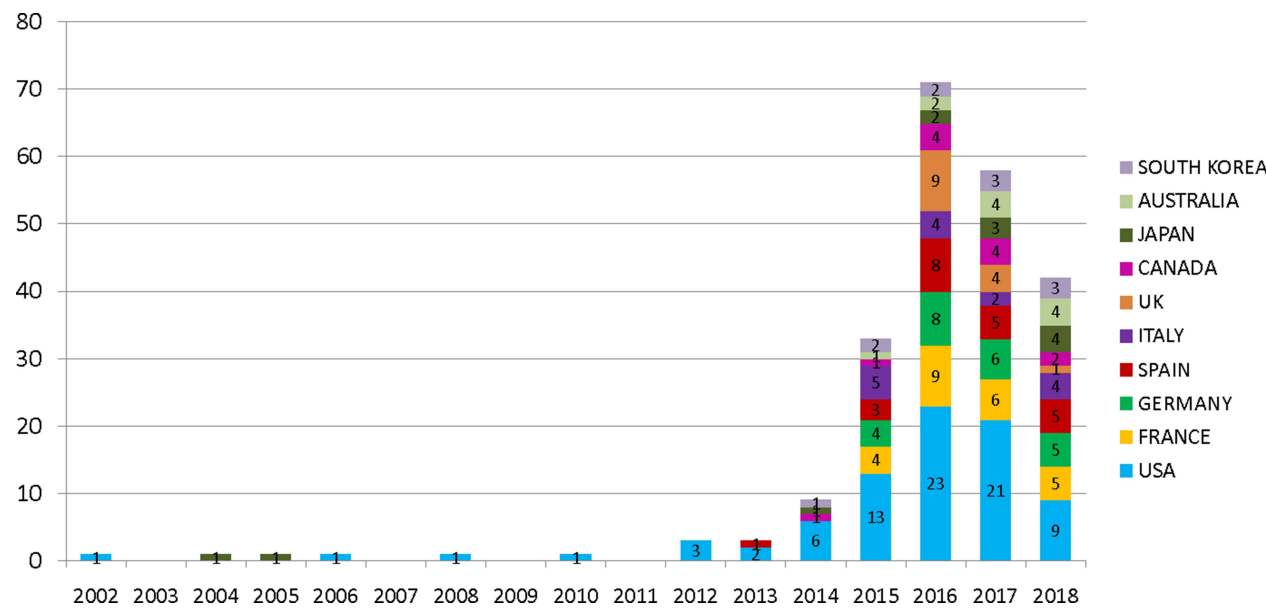

Figure 2 The no. of publications of different countries. 
Table I Journals Publishing the Top 100 Most Frequently Cited Articles

\begin{tabular}{|c|c|c|c|c|c|c|}
\hline Journal & $\begin{array}{l}\text { No. of } \\
\text { Articles }\end{array}$ & $\begin{array}{l}\text { Quartile in } \\
\text { Category }\end{array}$ & $\begin{array}{l}\text { Impact } \\
\text { Factor }\end{array}$ & H Index & $\begin{array}{l}\text { Citation } \\
\text { Count }\end{array}$ & Country \\
\hline New England Journal of Medicine & 16 & QI & 70.67 & 933 & 11,466 & United States \\
\hline Lancet Oncology & 9 & QI & 35.386 & 274 & 6032 & United States \\
\hline Journal of Clinical Oncology & 8 & QI & 28.245 & 494 & 2564 & United States \\
\hline Clinical Cancer Research & 8 & QI & 8.911 & 292 & 3643 & United States \\
\hline Annals of Oncology & 6 & QI & 14.196 & 210 & 1790 & England \\
\hline Lancet & 5 & QI & 59.102 & 700 & 2890 & England \\
\hline Science & 5 & QI & 41.037 & 1058 & $226 I$ & United States \\
\hline Nature & 3 & QI & 43.07 & 1096 & 2921 & England \\
\hline Journal of Thoracic Oncology & 3 & QI & 12.46 & 113 & 955 & United States \\
\hline Cell & 2 & QI & 36.216 & 705 & 673 & United States \\
\hline Nature Reviews Clinical Oncology & 2 & QI & 34.106 & 127 & 445 & England \\
\hline Cancer Discovery & 2 & QI & 26.37 & 119 & 1627 & United States \\
\hline JAMA Oncology & 2 & QI & 22.416 & 61 & 540 & United States \\
\hline Nature Communications & 2 & QI & 11.878 & 248 & 1044 & England \\
\hline European Journal of Cancer & 2 & QI & 6.68 & 193 & 831 & England \\
\hline Molecular Cancer Therapeutics & 2 & QI & 4.856 & 157 & 983 & United States \\
\hline Nature Reviews Cancer & 1 & QI & 51.848 & 396 & 713 & England \\
\hline Nature Reviews Immunology & I & QI & 44.019 & 351 & 875 & England \\
\hline Nature Medicine & I & QI & 30.641 & 497 & 2541 & England \\
\hline Cancer Cell & I & QI & 23.916 & 295 & 433 & United States \\
\hline Immunity & I & QI & 21.522 & 355 & 263 & United States \\
\hline Science Translational Medicine & I & QI & 17.161 & 168 & 628 & United States \\
\hline Blood & I & QI & 16.562 & 462 & 303 & United States \\
\hline Journal of Clinical Investigation & I & QI & 12.282 & 447 & 404 & United States \\
\hline Trends in Molecular Medicine & I & QI & 11.028 & 160 & 220 & England \\
\hline Journal for Immunotherapy of Cancer & I & QI & 8.676 & 36 & 282 & United States \\
\hline Cancer Treatment Reviews & 1 & QI & 8.332 & 116 & 305 & England \\
\hline $\begin{array}{l}\text { Journal of the National Comprehensive Cancer } \\
\text { Network }\end{array}$ & I & QI & 7.57 & 88 & 398 & United States \\
\hline British Journal of Cancer & I & QI & 5.416 & 212 & 303 & England \\
\hline Cancer Epidemiology Biomarkers \& Prevention & I & QI & 5.057 & 177 & 218 & United States \\
\hline Lung Cancer & I & QI & 4.599 & 114 & 220 & Netherlands \\
\hline International Immunology & I & QI & 4.168 & 124 & 237 & England \\
\hline Frontiers in Pharmacology & I & QI & 3.845 & 62 & 277 & Switzerland \\
\hline Neoplasia & 1 & Q2 & 3.836 & 115 & 371 & United States \\
\hline Laboratory Investigation & I & QI & 3.684 & 140 & 477 & United States \\
\hline Medical Oncology & I & QI & 3.252 & 64 & 352 & United States \\
\hline $\begin{array}{l}\text { American Journal of Clinical Oncology-cancer } \\
\text { Clinical Trials }\end{array}$ & I & Q2 & 3.015 & 72 & 436 & United States \\
\hline Clinical Therapeutics & I & Q2 & 2.935 & 123 & 228 & United States \\
\hline PLOS One & I & QI & 2.776 & 268 & 252 & United States \\
\hline
\end{tabular}

Hopkins Univ, 26\%) (Table 3). As shown in Figure 3, Johns Hopkins Univ, Yale Univ, Massachusetts Gen the cooperation among 28 institutions presented visually. Hosp, Beth Israel Deaconess Med, Sidney Kimmel In consociation with Mem Sloan Kettering Canc Ctr, the Comprehens Canc, Univ Michigan, Dana Farber Canc network about institutions showed that there were 27 Inst, Bristol Myers Squibb Co, Vanderbilt Univ, Yale institutions, which were Weill Cornell Med Coll, Canc Ctr, Univ Chicago, Merck\&Co Inc, Univ Columbia Univ, Univ Sydney, INSERM, Genetech Inc, Washington, Bristol Myers Squibb, Univ Texas MD Univ Calif Los Angeles, Mayo Clin, Harvard Univ, Anderson Canc Ct, Fox Chase Canc Ctr, Duke Univ, 
Table 2 Top 10 Authors Who Most Frequently Contributed to the Articles

\begin{tabular}{|c|c|c|c|c|c|c|}
\hline Rank & Author & $\begin{array}{l}\text { No. of } \\
\text { Articles }\end{array}$ & $\begin{array}{l}\text { As First } \\
\text { Author }\end{array}$ & $\begin{array}{l}\text { As Corresponding } \\
\text { Author }\end{array}$ & H Index & Affiliation \\
\hline 1 & Brahmer, JR & 11 & 2 & 2 & 69 & $\begin{array}{l}\text { Sidney Kimmel Comprehensive Cancer } \\
\text { Center }\end{array}$ \\
\hline 2 & Pardoll, DM & 10 & 0 & I & 126 & $\begin{array}{l}\text { Sidney Kimmel Comprehensive Cancer } \\
\text { Center }\end{array}$ \\
\hline 3 & Hellmann, MD & 10 & 2 & 4 & 56 & $\begin{array}{l}\text { Memorial Sloan Kettering Cancer Center } \\
\text { and Weill Cornell Medicine College }\end{array}$ \\
\hline 4 & Chen, LP & 9 & 0 & I & 52 & Yale Cancer Center \& Yale University \\
\hline 5 & Wolchok, JD & 9 & 0 & I & 114 & $\begin{array}{l}\text { Memorial Sloan Kettering Cancer Center } \\
\text { and Weill Cornell Medicine College }\end{array}$ \\
\hline 6 & Horn, L & 8 & 0 & 0 & 45 & Vanderbilt University \\
\hline 7 & Topalian, SL & 8 & 3 & 4 & 83 & $\begin{array}{l}\text { Sidney Kimmel Comprehensive Cancer } \\
\text { Center } \\
\text { and Johns Hopkins University }\end{array}$ \\
\hline 8 & Antonia, SJ & 8 & 3 & 2 & 57 & University of Florida \\
\hline 9 & Rizvi, NA & 7 & 2 & 2 & 55 & $\begin{array}{l}\text { Memorial Sloan Kettering Cancer Center } \\
\text { and Weill Cornell Medicine College }\end{array}$ \\
\hline 10 & Taube, JM & 7 & I & I & 46 & $\begin{array}{l}\text { Sidney Kimmel Comprehensive Cancer } \\
\text { Center }\end{array}$ \\
\hline
\end{tabular}

Ohio State Univ, Emory Univ. Moreover, three items were organized by cooperation, represented as different colors, red, green, and blue. Just as shown in the blue item, Mem Sloan Kettering Canc Ctr and Weill Cornell Med Coll were in the same item. Meanwhile, in the red item, Yale Can Ctr and Yale Univ were within their own

Table 3 The Top 10 Institutions Contributing to the 100 Most Frequently Cited Articles

\begin{tabular}{|l|l|l|}
\hline Institutions & Countries & $\begin{array}{l}\text { No.of } \\
\text { Publications }\end{array}$ \\
\hline Mem Sloan Kettering Canc Ctr & United States & 37 \\
Univ Texas MD Anderson Can & United States & 26 \\
Ctr & & \\
Johns Hopkins Univ & United States & 20 \\
Dana Farber Canc Inst & United States & 19 \\
Yale Univ & United States & 15 \\
Univ Paris Saclay & France & 15 \\
Massachusetts Gen Hosp & United States & 14 \\
Bristol Myers Squibb Co & United States & 13 \\
INSERM & France & 13 \\
Mayo Clin & United States & 10 \\
\hline
\end{tabular}

item. This suggested that different functional departments referred to the same institution.

\section{Researcher Coauthored Network Visualization}

To analyze the coauthorship network based on the information of the authors, setting the minimum number of coauthors at 2 , and then, 85 authors meet the thresholds of the total 843 authors. In network visualization shown in Figure 4, each circle and label represented an author, and the size of circles represented the frequency of occurrence. The larger the circle was, the more frequently the circle-represented body appeared. As shown, the circles representing brahmer, jr., pardoll, dm., hellmann, md., chen, 1., wolchok, jd., etc., mainly the same as Table 2 shown were larger than others, which indicated that they contributed to more collaborating articles. And then, the more relevant the authors were, the closer the circles were located, ${ }^{13}$ such as chen, 1 and sznol, mario. Lines linking two circles indicated that the two authors had appeared in the same publication. Therefore, connection lines derived from the former five authors were denser and thicker, indicating their closer relationship. Seven items were developed, which were mainly due to their contributions to the same publication. 
\& VOSviewer

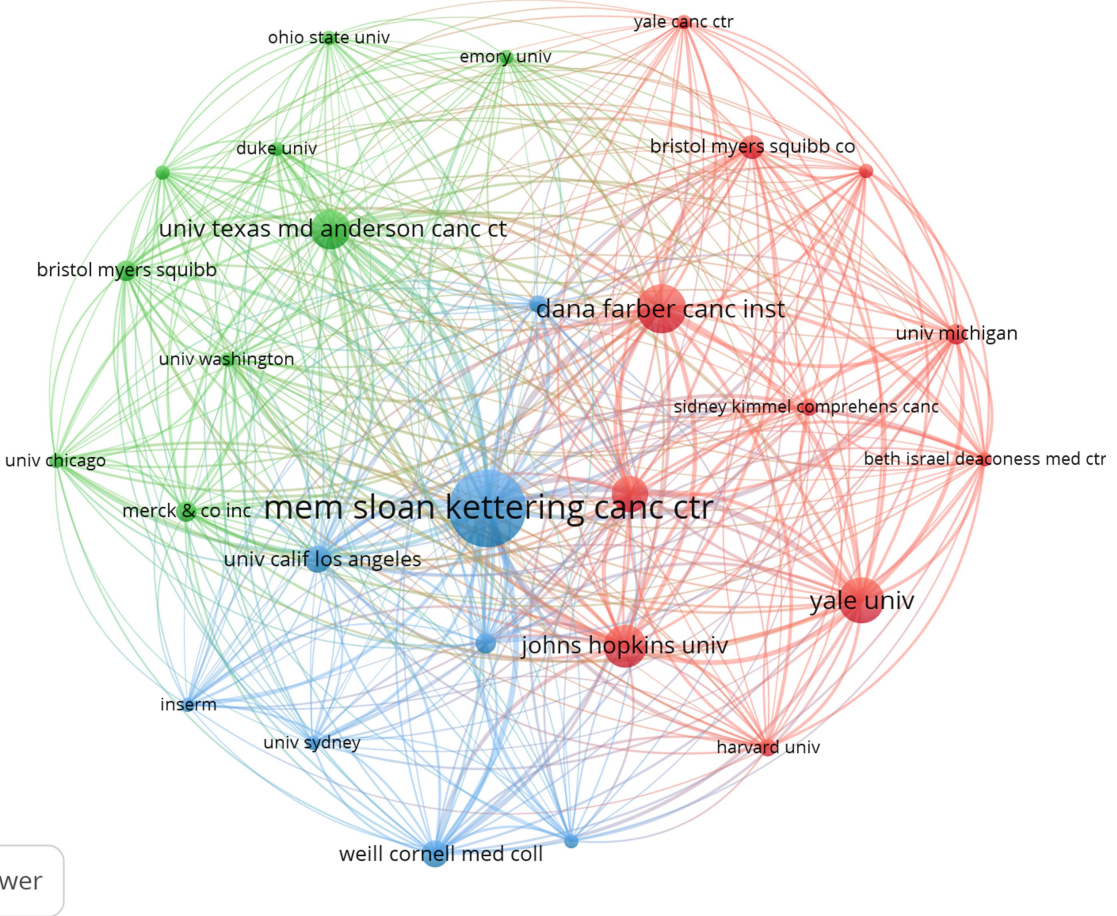

Figure 3 Institutions co-operation network visualization of the 100 most cited publications.

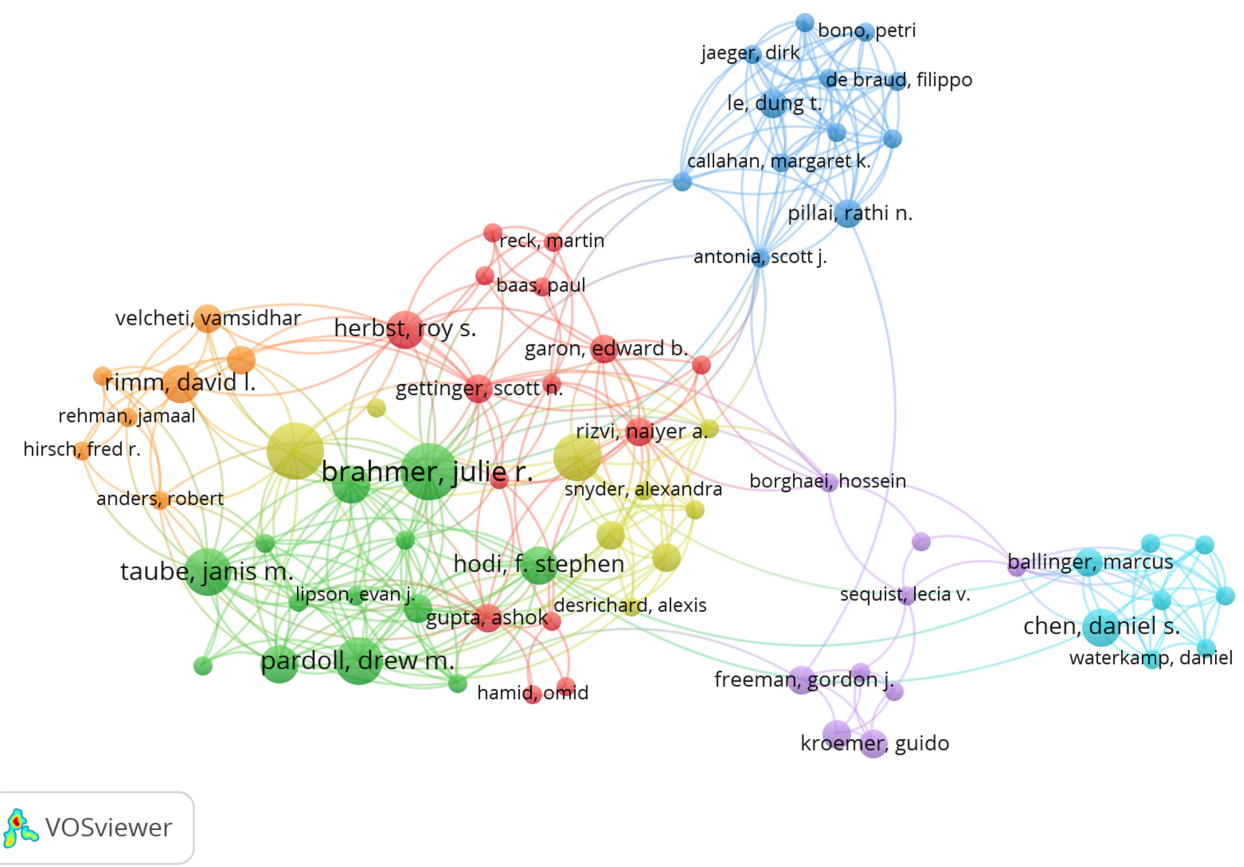

Figure 4 Authors coauthorship network visualization of the 100 most frequently cited publications.

\section{Keyword Co-Occurrence Network} Visualization

VOSviewer software was also used to draw the network of keywords co-occurrence, setting the minimum number of occurrences at 10. As shown in Figure 5, for the circles, larger circles representing keywords such as immunotherapy, nivolumab, cell lung-cancer, safety, and docetaxel, indicated that these keywords appeared more frequently. Immunotherapy, the origin of the anti-PD-1/PD-L1 therapy, has been extensively studied, and linked to $42(87.5 \%)$ keywords. 


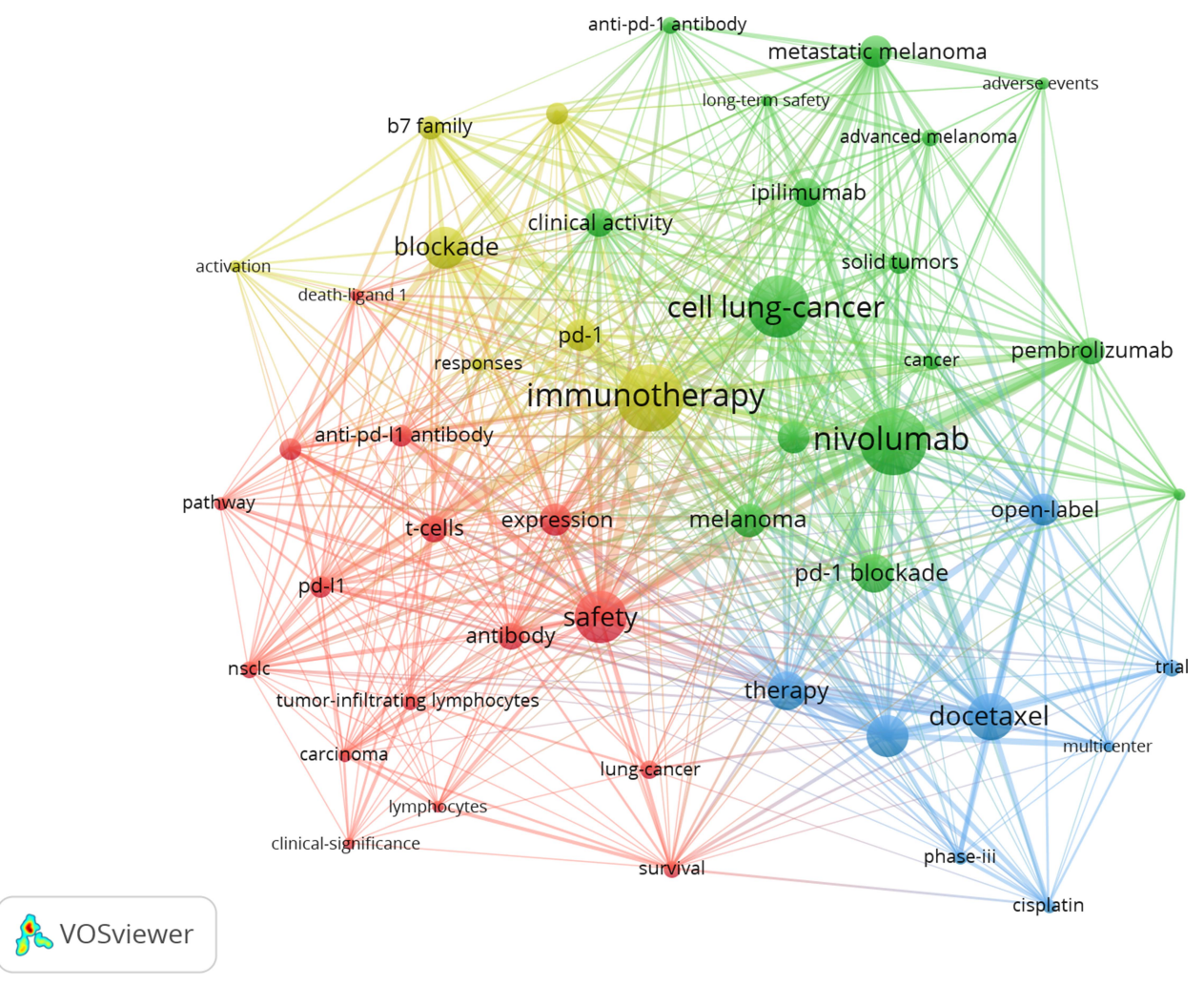

Figure 5 Keywords co-occurrence network visualization of the 100 most frequently cited publications.

In network visualization, circles adopting various colors in graph represented different clusters. Four clusters were separated based on their association. In cluster 1 , which was colored red, most of them were associated with the expression of PD-1, PD-L1 in lymphocytes, tumor-infiltrating lymphocytes, or T-cells of the patients with lung cancer, and the survival and safety of the treatment. In cluster 2, which was colored green, a portion of them were associated with the specific PD-1 blockade, such as pembrolizumab, and another drug nivolumab in combination with ipilimumab, while others were mainly associated with the specific patient, such as patients with solid tumors, cell lung cancer, melanoma, advanced melanoma, metastatic melanoma, and untreated melanoma. In cluster 3, which was colored blue, a portion of them were associated with the levels of the trials, such as multicenter, and phaseiii, while others were mainly associated with the specific second-line chemotherapy drugs, such as docetaxel, and cisplatin. In cluster 4, which was colored yellow, most of them were associated with the biological effect of immunotherapy, such as activation, antitumor immunity, and responses.

\section{Discussion}

Bibliometric analysis or bibliometric methods, which originating from the field of library and information and motivating by the fast development of computers and the Internet, are now firmly established as scientific specialties and act as an integral part of research evaluation methodology especially within the scientific and applied fields. ${ }^{14,15}$ The methods are used increasingly in analyzing various aspects of science. In the present study, the top 100 most frequently cited publications in the field of anti-PD -1/PD-L1 therapy for lung cancer were retrieved to perform a bibliometric analysis.

Since the first article published in 2002, it had been cited for 2541 times, which provided implications for the design of $\mathrm{T}$ cell-based cancer immunotherapy mediated by one or more receptors other than PD-1. ${ }^{16}$ The study concerning the anti-PD-1/PD-L1 therapy for lung cancer was still an emerging approach, which manifested as a large amount of scientific works and had attracted more attention in recent years. Among the top 100 cited articles, albeit the totally cited times ranged from 218 to 6,248 , it was different sorting by the cited frequencies per year. In total, $33 \%$ of the publications were cited less than 100 
times per year, while $8 \%$ were cited less than 50 times per year. In 2019, a clinical research was cited for 138 times, which was more than 100 times per year, ${ }^{12}$ followed by a review and two articles cited 89,80 , and 50 times, which were cited no less than 50 times per year. ${ }^{17-19}$ The comparison suggested that the index cited times per year might be more precise to assess the academic influence of the publications. No matter what, the frequently cited publications, including the articles in 2019, which had higher levels of evidence, were more apt to be cited. Most of the articles about clinical research included randomized, large scale samples, and especially registered at clinical trials.gov. ${ }^{17,19,20}$

Among the 39 journals, except for three journals rating at Q2, Neoplasia, American Journal of Clinical OncologyCancer Clinical Trials, and ClinicalTherapeutics, other journals were all rating at Q1. More than half of the articles were published in seven journals, including New England Journal of Medicine, Lancet Oncology, Journal of Clinical Oncology, Clinical Cancer Research, Annals of Oncology, Lancet, and Science. Three of them were comprehensive journals, while other four focused on the research of oncology. The New England Journal of Medicine, which published most articles (16\%) and possessed the highest impact factor in the present study, had published the top three cited articles, ${ }^{12,21,22}$ with the total citation reaching 13,835 times. For the three articles, they were associated with the safety, activity of anti-PD1 antibody in the patients with NSCLC, which suggested that the clinical outcome might be more attractive than the preclinical research about the mechanism of the PD-1 and PD-L1 checkpoint signaling inhibition. For the journals, all of them are peer-reviewed journals, and the publications are only possible because the arciles are high quality, including novel or at least prospective design, use of standardised definitions. ${ }^{23}$ Apart from the above predefined criteria, the sample size and type of patient population also matter. Setting an example, research about the treatment of glioblastoma published in New England Journal of Medicine, which was cited more than 10,000 times, included a total of 573 patients from 85 centers. $^{24}$

According to the data of GLOBOCAN 2018, the highest incidence rates among men are observed in Micronesia/ Polynesia, in Eastern Asia (rates are more than 40 per 100,000 in China, Japan, and the Republic of Korea), and in much of Europe, especially in Eastern Europe. ${ }^{1}$ The incidence rate of lung cancer in Europe were much more than that in America. Nonetheless, most of the articles were from United States of America, European countries, and some developing Asian countries. The most significant reason is that the United States and Europe are pioneers in cancer immunotherapy. The first related article was published by an institution in the USA about the development a novel treatment paradigm. Moreover, the best-known pharmaceutical factory, Bristol-Myers Squibb, as the producer of the anti-PD-1/PD-L1 agent Nivolumab $\left(\mathrm{OPDIVO}^{\circledR}\right)$ is located in the USA. Another reason is that Nivolumab (OPDIVO ${ }^{\circledR}$ ) was approved by the FDA earlier than in other countries.

The statistics about the authors and the institutions suggested that the United States was the major country contributing to the studies in the field of immunotherapy for lung cancer. The top 10 authors carried influence in the academic world, presented as their higher H-index. They were all did their research at institutions in the USA, while four of them work in Sidney Kimmel Comprehensive Cancer Center, and three in Memorial Sloan Kettering Cancer Center \& Weill Cornell Medicine College. In addition, the top 10 institutions showed that $80 \%$ of them were located in USA, while the rest of them were in France. In network visualization of the institutions, the most important institution was Memorial Sloan Kettering Cancer Center, which co-operated with more than 25 native institutions, and two foreign institutions, INSERM, and Univ Paris Saclay. The cooperation among different institutions or different departments might be beneficial to data collection and to decrease the racial differences in the studies. $^{24,25}$ More than that, the studies with big scale samples cooperated with multiple centers, another reason was that some authors were employed by different institutions.

In the coauthored network, outstanding researchers in this field were relatively concentrated, moreover, less of them had published more articles. Researchers such as Brahmer JR, Pardoll DM, and Taube JM from Sidney Kimmel Comprehensive Cancer Center had published more articles and they were in close contact with each other in the field. The reason may be that the chance of working together among them is much more than that of others due to they work in the same situation, which is beneficial to discuss the research designing, samples collecting, etc. The reason of close contact for other authors is probably due to multi-center research, even some international cooperations. ${ }^{22}$ 
In the visualization of co-occurrence based on all keywords, the top 100 highly cited articles covered various aspects of anti-PD-1/PD-L1 therapy for lung cancer, such as the drugs used as immunotherapy, chemotherapy, and cell lung-cancer, for example, nivolumab, docetaxel, ${ }^{22,26}$ another crucial topic in the clinical research, therapy, blockade, safety, and clinical activity, ${ }^{12}$ and expression, blockage, b7 family, and CTLA-4 blockade, which concerned in the type of anti-PD1/PD-L1 and other immune checkpoint blockade inhibitors. ${ }^{27}$ In recent years, a variety of immunotherapies have been approved for clinical lung cancer, especially in NSCLC treatment.

In network visualization, the links between different keywords were also analyzed. The origin of the present study was to explore the acknowledge of immunotherapy for lung cancer, 42 keywords $(87.5 \%)$ linked to immunotherapy. Based on the total link strength, the linking between immunotherapy and nivolumab, cell lung-cancer, safety, chemotherapy, docetaxol, blockage, b7 family, pd$1, \mathrm{pd}-\mathrm{L} 1$, expression, were ranked in sequence. Just as shown, the most important drugs as anti-PD1/PD-L1 treatment for cell lung-cancer were nivolumab and docetaxol. Nivolumab (OPDIVO ${ }^{\circledR}$, Bristol-Myers Squibb), as PD-1 inhibitors, has been approved by the FDA and EMA as second-line therapy in metastatic NSCLC irrespectively of PD-L1 expression. The biological efficacy of Nivolumab on treatment for NSCLC was evident, however, development of immune-related adverse events was associated with survival outcomes of nivolumab treatment in patients with advanced or recurrent NSCLC, and then early recognition and proper management of immunerelated adverse events are important to maximize the therapeutic benefit of immune-checkpoint inhibitors in patients with NSCLC. ${ }^{28}$ Another strategy to improve the efficacy of Nivolumab monotherapy in lung cancer is to combine it with ipilimumab, both in SCLC and NSCLC. ${ }^{29,30}$ Among patients with advanced NSCLC, the response rate was better with nivolumab plus ipilimumab than with nivolumab monotherapy, more than that, the first-line treatment with nivolumab plus ipilimumab resulted in a longer duration of overall survival than did chemotherapy in patients with late stage or recurrent NSCLC. The microtubule inhibitor, docetaxol (Taxotere ${ }^{\circledR}$, Sanofi) was evaluated as second-line treatment for NSCLC. Considering that the checkpoint inhibitors in combination with chemotherapy are going to become the standard-of-care for first-line therapy, the combination of antiangiogenic drugs, such as nintedanib or ramucirumab, plus docetaxel, may become the new options for second-line therapy of nonsquamous NSCLC patients. ${ }^{31}$ Docetaxel plus ramucirumab was associated with a significant improvement in overall survival and progression-free survival, relative to docetaxel, regardless of patient type. In other words, the efficacy of combined therapy of Docetaxel plus Ramucirumab showed irrespective of PD-L1 expression in NSCLC patients. ${ }^{32}$ While Docetaxel plus nintedanib showed similar efficacy to docetaxel plus ramucirumab in the nonsquamous populations. ${ }^{33}$

The management of lung cancer, especially NSCLC, has shifted dramatically in recent decades with an increase in the understanding of the biological characteristics and with the development of precision medicine. Different immune checkpoint inhibitors: anti PD-1 (nivolumab and pembrolizumab) and PD-L1 (atezolizumab, durvalumab, and avelumab) medications were used in the patients with advanced lung cancer. Amount of data on the combinations of PD-1/PD-L1 inhibitors with other therapies, such as immunotherapy, targeted therapy and chemotherapy, have been addressed, involving in the toxicity profile, and the efficacy of these agents. Immunotherapy has definitely altered the paradigm on the treatment of lung cancer, and the future is promising for the benefit of patients.

In general, the top 100 most frequently cited articles on anti-PD-1/PD-L1 therapy for lung cancer were analyzed via bibliometrics combined with network visualization analysis, which yielding up the advantage of identifying and analyzing the characteristics and changes in the intellectual structures of a special topic. Most of the scientific works were published after the year 2000 on top journals, such as the New England Journal of Medicine. The United States acted as the pioneer and kept its leadership in this new field of research. The most important key words, such as immunotherapy, nivolumab, cell lung-cancer, safety, and docetaxel, were stressed. To sum up, the most important reason for the frequent citation of the publications might include the high quality journals, research with high quality evidence, and influential authors and institutions. Most importantly, this topic has attracted much more attention globally in recent years, suggesting that numerous studies on anti-PD-1/PD-L1 therapy for lung cancer may arise in the future.

\section{Disclosure}

The authors report no conflicts of interest for this work. 


\section{References}

1. Bray F, Ferlay J, Soerjomataram I, et al. Global cancer statistics 2018: GLOBOCAN estimates of incidence and mortality worldwide for 36 cancers in 185 countries. CA Cancer J Clin. 2018;68 (6):394-424. doi:10.3322/caac. 21492

2. Goodwin PJ, Shepherd FA. Economic issues in lung cancer: a review.. J Clin Oncol. 1998;16(12):3900-3912. doi:10.1200/JCO.19 98.16.12.3900

3. Urman A, Hosgood HD. Curbing the burden of lung cancer. Front Med. 2016;10(2):228-232. doi:10.1007/s11684-016-0447-x

4. Siegel RL, Miller KD, Jemal A. Cancer statistics, 2020. CA Cancer J Clin. 2020;70(1):7-30. doi:10.3322/caac. 21590

5. Kennedy LB, Salama AKS A. A review of cancer immunotherapy toxicity. CA Cancer J Clin. 2020;70(2):86-104. doi:10.3322/ caac. 21596

6. Herbst RS, Morgensztern D, Boshoff C. The biology and management of non-small cell lung cancer. Nature. 2018;553 (7689):446-454. doi:10.1038/nature25183

7. Ragavan M, Das M. Systemic Therapy of Extensive Stage Small Cell Lung Cancer in the Era of Immunotherapy. Curr Treat Options Oncol. 2020;21(8):64-77. doi:10.1007/s11864-020-00762-8

8. Lim SM, Hong MH, Kim HR. Immunotherapy for Non-small Cell Lung Cancer: current Landscape and Future Perspectives. Immune Netw. 2020;20(1):e10. doi:10.4110/in.2020.20.e10

9. Horn L, Mansfield AS, Szczęsna A, et al. First-Line Atezolizumab plus Chemotherapy in Extensive-Stage Small-Cell Lung Cancer. $N$ Engl J Med. 2018;379(23):2220-2229. doi:10.1056/NEJMoa18 09064

10. Saab S, Zalzale H, Rahal Z, et al. Insights Into Lung Cancer Immune-Based Biology, Prevention, and Treatment. Front Immunol. 2020;11:159. doi:10.3389/fimmu.2020.00159

11. Rizvi NA, Hellmann MD, Snyder A, et al. Cancer immunology. Mutational landscape determines sensitivity to PD-1 blockade in non-small cell lung cancer. Science. 2015;348(6230):124-128. doi:10.1126/science.aaa1348

12. Topalian SL, Hodi FS, Brahmer JR, et al. Safety, Activity, and Immune Correlates of Anti-PD-1 Antibody in Cancer. $N$ Engl $J$ Med. 2012;366(26):2443-2454. doi:10.1056/NEJMoa1200690

13. van Eck NJ, Waltman L. Software survey: vOSviewer, a computer program for bibliometric mapping. Scientometrics. 2010;84 (2):523-538. doi:10.1007/s11192-009-0146-3

14. Bar-Ilan J. Informetrics at the beginning of the 21 st century-a review. $J$ Informetr. 2008;2(1):1-52. doi:10.1016/j.joi.2007.11.001

15. Ellegaard O, Wallin JA. The bibliometric analysis of scholarly production: how great is the impact? Scientometrics. 2015;105 (3):1809-1831. doi:10.1007/s11192-015-1645-z

16. Dong H, Strome SE, Salomao DR, et al. Tumor-associated B7-H1 promotes T-cell apoptosis: A potential mechanism of immune evasion. Nat Med. 2002;8(8):793-800. doi:10.1038/nm730

17. Mok TSK, Wu Y-L, Kudaba I, et al. Pembrolizumab versus chemotherapy for previously untreated, PD-L1-expressing, locally advanced or metastatic non-small-cell lung cancer (KEYNOTE-042): a randomised, open-label, controlled, Phase 3 trial. Lancet. 2019;393(10183):1819-1830. doi:10.1016/S01406736(18)32409-7

18. Chan TA, Yarchoan M, Jaffee E, et al. Development of tumor mutation burden as an immunotherapy biomarker: utility for the oncology clinic. Ann Oncol. 2019;30(1):44-56. doi:10.1093/annonc/mdy495
19. Reck M, Rodríguez-Abreu D, Robinson AG, et al. Updated Analysis of KEYNOTE-024: pembrolizumab versus platinum-based chemotherapy for advanced non-small-cell lung cancer with pd-11 tumor proportion score of $50 \%$ or greater. J Clin Oncol. 2019;37 (7):537-546. doi:10.1200/JCO.18.00149

20. Ott PA, Bang Y-J, Piha-Paul SA, et al. T-cell-inflamed geneexpression profile, programmed death ligand 1 expression, and tumor mutational burden predict efficacy in patients treated with pembrolizumab across 20 cancers: KEYNOTE-028. J Clin Oncol. 2019;37(4):318-327. doi:10.1200/JCO.2018.78.2276

21. Brahmer JR, Tykodi SS, Chow LQM, et al. Safety and Activity of Anti-PD-L1 Antibody in Patients with Advanced Cancer. $N$ Engl J Med. 2012;366(26):2455-2465. doi:10.1056/NEJMoa1200694

22. Borghaei H, Paz-Ares L, Horn L, et al. Nivolumab versus Docetaxel in Advanced Nonsquamous Non-Small-Cell Lung Cancer. $N$ Engl $J$ Med. 2015;373(17):1627-1639. doi:10.1056/NEJMoa1507643

23. Allegranzi B, Bagheri Nejad S, Combescure C, et al. Burden of endemic health-care-associated infection in developing countries: systematic review and meta-analysis. Lancet. 2011;377 (9761):228-241. doi:10.1016/S0140-6736(10)61458-4

24. Stupp R, Mason WP, van den Bent MJ, et al. Radiotherapy plus concomitant and adjuvant temozolomide for glioblastoma. $N$ Engl J Med. 2005;352(10):987-996. doi:10.1056/NEJMoa043330

25. Vanbutsele G, Pardon K, Van Belle S, et al. Effect of early and systematic integration of palliative care in patients with advanced cancer: a randomised controlled trial. Lancet Oncol. 2018;19 (3):394-404. doi:10.1016/S1470-2045(18)30060-3

26. Brahmer J, Reckamp KL, Baas P, et al. Nivolumab versus docetaxel in advanced squamous-cell non-small-cell lung cancer. $N$ Engl J Med. 2015;373(2):123-135. doi:10.1056/NEJMoa1504627

27. Pardoll DM. The blockade of immune checkpoints in cancer immunotherapy. Nat Rev Cancer. 2012;12(4):252-264. doi:10.1038/ $\operatorname{nrc} 3239$

28. Haratani K, Hayashi H, Chiba Y, et al. Association of immune-related adverse events with nivolumab efficacy in non-small-cell lung cancer. JAMA Oncol. 2018;4(3):374-378. doi:10.1001/jamaoncol.20 17.2925

29. Hellmann MD, Callahan MK, Awad MM, et al. Tumor mutational burden and efficacy of nivolumab monotherapy and in combination with ipilimumab in small-cell lung cancer. Cancer Cell. 2018;33 (5):853-861.e4. doi:10.1016/j.ccell.2018.04.001

30. Hellmann MD, Paz-Ares L, Bernabe Caro R, et al. Nivolumab plus Ipilimumab in advanced non-small-cell lung cancer. $N$ Engl J Med. 2019;381(21):2020-2031. doi:10.1056/NEJMoa1910231

31. Rossi A. New options for combination therapy for advanced non-squamous NSCLC. Expert Rev Respir Med. 2019;13 (11):1095-1107. doi:10.1080/17476348.2019.1667233

32. Yoshimura A, Yamada T, Okuma Y, et al. Retrospective analysis of docetaxel in combination with ramucirumab for previously treated non-small cell lung cancer patients. Transl Lung Cancer Res. 2019;8 (4):450-460. doi:10.21037/tlcr.2019.08.07

33. Vickers AD, Winfree KB, Cuyun Carter G, et al. Relative efficacy of interventions in the treatment of second-line non-small cell lung cancer: a systematic review and network meta-analysis. BMC Cancer. 2019;19(1):353. doi:10.1186/s12885-019-5569-5 


\section{Publish your work in this journal}

Cancer Management and Research is an international, peer-reviewed open access journal focusing on cancer research and the optimal use of preventative and integrated treatment interventions to achieve improved outcomes, enhanced survival and quality of life for the cancer patient.

The manuscript management system is completely online and includes a very quick and fair peer-review system, which is all easy to use. Visit http://www.dovepress.com/testimonials.php to read real quotes from published authors. 